\title{
EDUCAÇÃO EM SAÚDE COM GESTANTES INTERMEDIADAS PELO GESTAR
}

\author{
Victória Friedrich Costa ${ }^{1}$; Leandro da Silva de Medeiros 2 ; Giovana Luiza \\ Rossato ${ }^{3}$; Camila Cioquetta Pereira ${ }^{4}$; Eduarda Dorneles da Silva ${ }^{5}$ Andressa \\ Caetano da Veiga ${ }^{6}$; Dirce Stein Backes ${ }^{7}$
}

\section{RESUMO}

Objetivo: Relatar a experiência de ações de educação em saúde com gestantes intermediadas pelo grupo de gestantes - GESTAR. Metodologia: Trata-se de um relato de experiências vivenciadas na realização das ações de saúde para gestantes pelos estudantes de Enfermagem de uma instituição comunitária do interior do estado do Rio Grande do Sul, no período de dezembro de 2020 a setembro de 2021. Resultados: Na perspectiva de humanizar e qualificar a atenção em saúde, esses encontros possibilitam compartilhar saberes, práticas educativas, procurando articular vários campos de conhecimentos necessários no cuidado integral à mulher e ser um instrumento de garantia dos direitos nas ações de saúde, na liberdade de escolha, apoio e incentivo durante o processo gravídico-puerperal. Conclusão: A realização de grupos de forma remota tem relevância no fortalecimento de vínculos entre as gestantes, seus grupos sociais e profissionais, potencializando o cuidado da mulher e dos processos que envolvem a gestação.

Palavras-chave: Educação em Saúde; Gravidez; Saúde Materno-Infantil.

Eixo Temático: Atenção Integral e Promoção à Saúde - AIPS.

\section{INTRODUÇÃO}

De acordo com Alves e Bezerra (2020) a gravidez é um período que provoca várias mudanças físicas, emocionais e sociais. Ainda, essas alterações geram

\footnotetext{
${ }^{1}$ Acadêmica do Curso de Enfermagem da Universidade Franciscana (UFN). Membro do GESTAR. E-mail: victoriafriedric27@gmail.com

2 Acadêmico do Curso de Enfermagem da Universidade Franciscana (UFN). Bolsista PPSUS/FAPERGS. E-mail: leandro.medeiros@ufn.edu.br

${ }^{3}$ Acadêmica do Curso de Enfermagem da Universidade Franciscana (UFN). Bolsista PROBIC/UFN. E-mail: rossatogiovana@gmail.com

${ }^{4}$ Acadêmica do Curso de Enfermagem da Universidade Franciscana (UFN). Membro do GESTAR. E-mail: camicioquetta@gmail.com

${ }^{5}$ Acadêmica do Curso de Enfermagem da Universidade Franciscana (UFN). Membro do GESTAR. E-mail: dorneleseduarda29@gmail.com

${ }^{6}$ Fisioterapeuta. Mestranda do Curso de Mestrado Profissional em Saúde Materno Infantil da Universidade Franciscana (UFN). E-mail: Andressacveiga@gmail.com

${ }^{7}$ Enfermeira. Doutora em Enfermagem. Coordenado do Mestrado Profissional em Saúde Materno Infantil da Universidade Franciscana (UFN). E-mail: backesdirce@ufn.edu.br
} 
sentimentos, como ansiedade, medo, angústia, dúvida, fantasia, entre outros, o que exige uma série de adaptações tanto da mulher como de seu parceiro (ALVES; BEZERRA, 2020).

Nesse período de mudanças, o casal necessita obter informações e orientações que auxiliem no desenvolvimento da gestação e no puerpério, sendo portando um período impar para a realização de ações educativas. De acordo com Vasconcelos et al (2016), dentre as ações de saúde temos o forte aliado a educação em saúde, que visa desenvolver um sentido de responsabilidade, como indivíduo, membro de uma família e de uma comunidade, para com a saúde, tanto individual como coletivamente.

A abordagem em grupo propicia ambiente para a promoção da saúde pelo processo de ensinar-aprender, constituindo-se num método privilegiado de investigação e intervenção. A gestação é um período importante para realização de ações educativas, pois propicia um intercâmbio de vivências e conhecimentos. Assim, o grupo de gestantes pode ser considerado uma forma de promover a compreensão do processo de gestação.

O grupo de orientação às gestantes caracteriza-se por uma ação educativa com o objetivo de facilitar a disseminação da informação e apropriação do conhecimento e favorecer a troca de experiências e é de grande importância para as futuras mães conhecerem todo o processo que envolve o ciclo gravídico-puerperal (GUERREIRO et al., 2012).

O grupo de gestantes possibilita uma espécie de filtro de práticas, onde, através de conversas e discussões, se exerce uma troca de conhecimentos/experiências e se visualiza o porquê de utilizar ou não determinada prática. Funciona como uma possibilidade diferenciada para o enfrentamento das mudanças decorrentes da gestação, uma vez que possui um cunho terapêutico e informativo para gestantes e acompanhantes (NUNES et al., 2017).

Em consonância com o exposto, o Grupo GESTAR, tem por finalidade fomentar discussões teórico-práticas que contribuam para a qualificação da rede de atenção à saúde materno-infantil. Sendo assim, este trabalho visa relatar a 
experiência de ações de educação em saúde com gestantes intermediadas pelo grupo GESTAR.

\section{METODOLOGIA}

Trata-se de um relato de experiências vivenciadas na realização das ações de saúde para gestantes pelos estudantes de Enfermagem de uma instituição comunitária do interior do estado do Rio Grande do Sul, no período de dezembro de 2020 a setembro de 2021.

O grupo GESTAR ocorre em parceria com residentes em Enfermagem Obstétrica e mestrandos do Curso de Mestrado Profissional em Sáude Materno Infantil da mesma instituição. O mesmo, anterior à pandemia, utilizava os espaços da IES para os encontros quinzenais e frente à COVID-19 os encontros ocorrem de forma remota por meio das mídias sociais Instagram e Facebook.

Conforme cronograma previamente organizado pelo grupo são abordados temas quanto aos cuidados com a mulher e o bebê no puerpério, direitos da gestante, puérpera e família, mitos e verdades sobre gestação e amamentação, o trabalho ocupacional da gestante em período de pandemia, sexualidade no pósparto, paternidade e cuidado e saúde mental perinatal, além de trocas de experiências entre as grávidas, profissionais, estudantes, residentes e mestrandos (Figura 1).

Figura 1 - Cards dos encontros temáticos

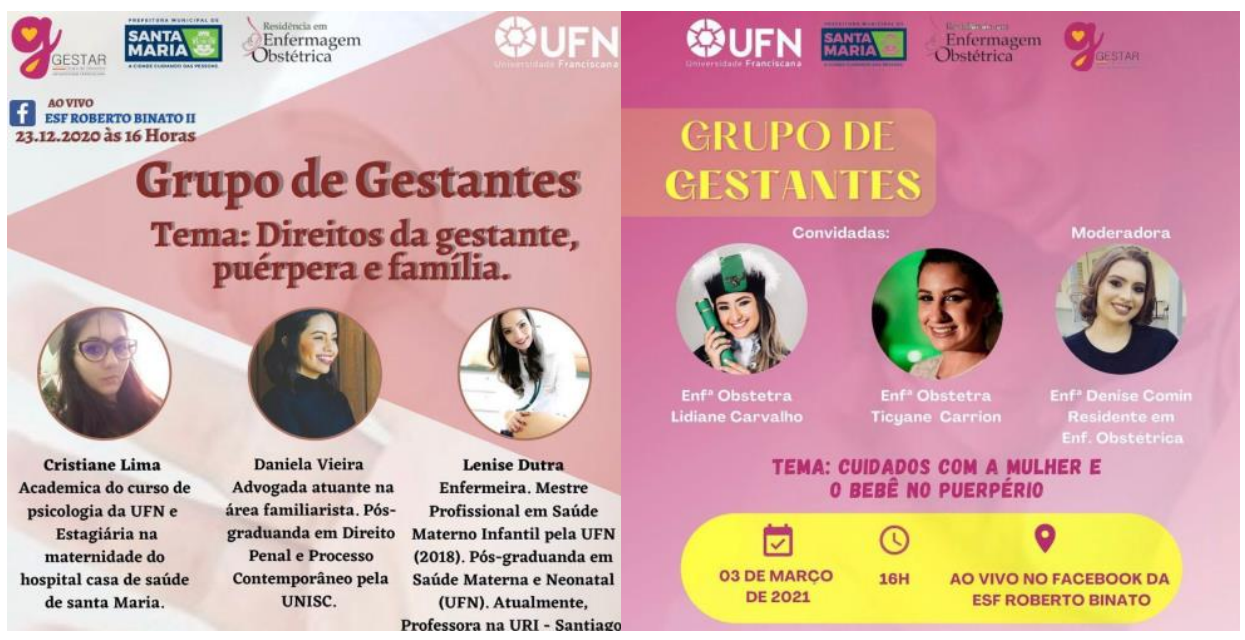


EDUGAÇÃO, SAÚDE ETECNOLOGIA

26 A 28 DE OUTUBRO DE 2021

SOLCक्ष०

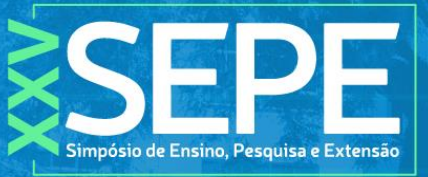

QuFN
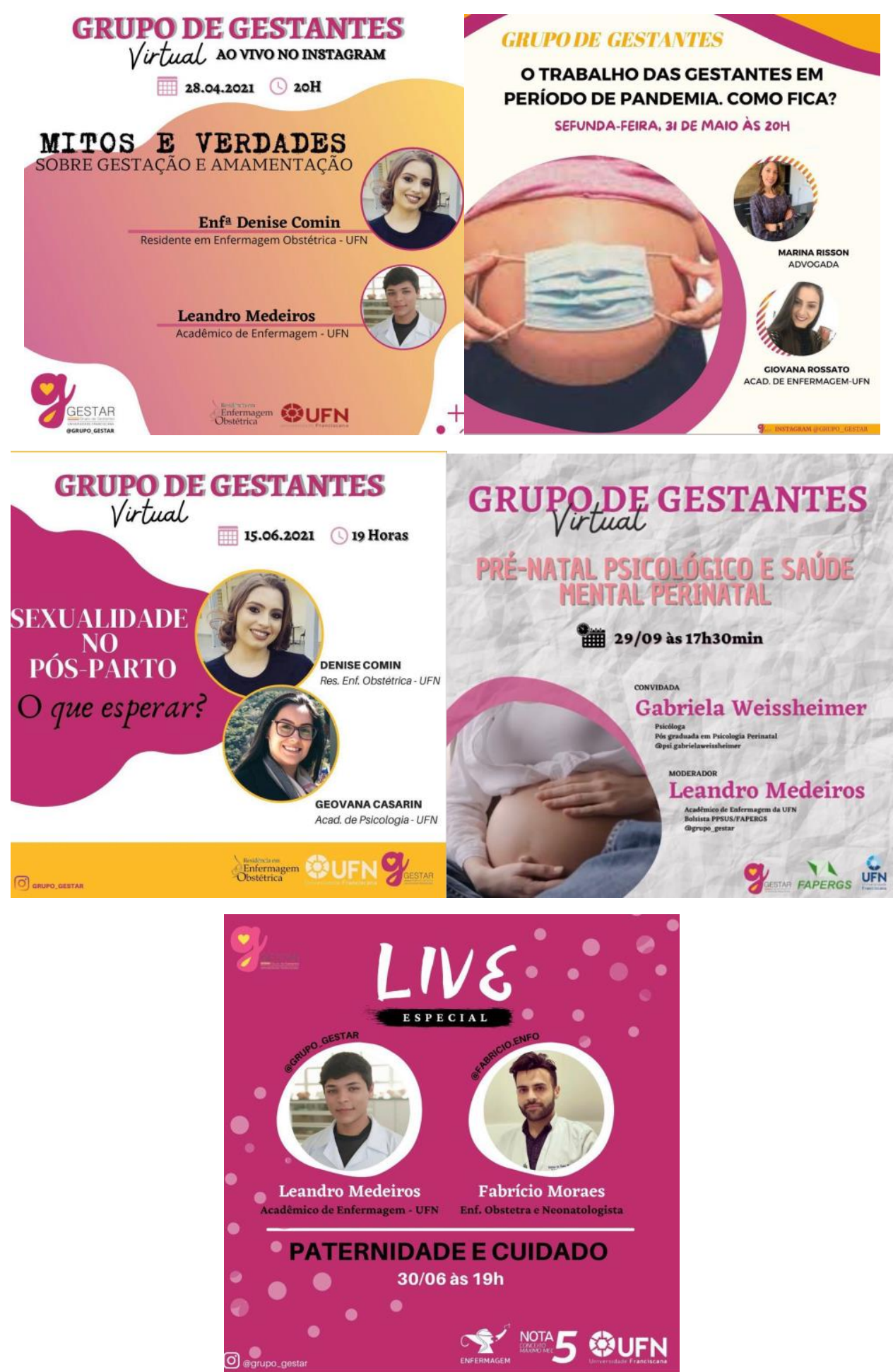

Fonte: Autores

Ressalta-se que o estudo faz parte de uma pesquisa maior intitulado "Qualificação da rede de atenção à saúde materno-infantil na região central do Rio 
Grande do Sul" que tem parecer favorável do Comitê de Ética em Pesquisa (CEP) sob o número de protocolo: 4.253.922.

\section{RESULTADOS E DISCUSSÕES}

Os documentos oficiais do Ministério da Saúde sobre a assistência à mulher sugerem, normatizam e estabelecem a educação em saúde no pré-natal. Eles estão respaldados por estudos científicos que comprovam sua efetividade na promoção da saúde durante o evento reprodutivo e dá subsídios para a continuidade da implementação das ações educativas no âmbito do SUS (CASTIGLIONI et al., 2020).

As abordagens ocorreram no grupo de gestantes - GESTAR. O grupo de gestantes já era realizado quinzenalmente na IES, após a pandemia os encontros ocorreram de forma remota com o objetivo de fortalecermos o vínculo, principalmente com os profissionais dos serviços de saúde, realizando a promoção da saúde e prevenção de doenças e agravos, bem como, realizar a troca de experiências entre as gestantes e as profissionais a partir da proposta de ações multiprofissionais, ações como estas possibilitam o empoderamento das gestantes quanto ao processo gravídico-puerperal.

Arrais, Mourão e Fragalle (2014) afirmam que a perspectiva multiprofissional viabiliza maior integração, viabilizando o acesso a questões que ultrapassam a lógica médica o que potencializa maior autonomia e autocuidado no processo gravídico e pós-parto. Nesse sentido, ainda, consideram que a troca de experiências entre as gestantes fortalece a rede de apoio social que em muitas situações mostrase fragilizada pelas diversas mudanças corporais e psicossociais que envolvem este momento da vida da mulher.

$\mathrm{Na}$ perspectiva de humanizar e qualificar a atenção em saúde, esses encontros possibilitam compartilhar saberes, práticas educativas, procurando articular vários campos de conhecimentos necessários no cuidado integral à mulher e ser um instrumento de garantia dos direitos nas ações de saúde, na liberdade de escolha, apoio e incentivo durante o processo do parto. 
Nas atividades desenvolvidas, as gestantes puderam compreender os fenômenos corporais e psicoemocionais envolvidos no processo gestacional, bem como, sobre a promoção de um estilo de vida ativo, hábitos saudáveis, etapas da gestação e do parto, direitos das gestantes, orientações sobre o parto humanizado, potencializando a prevenção da violência obstétrica, importância da amamentação e cuidados com as mamas, cuidados e valorização da saúde mental e uso de substâncias psicoativas.

Diferentes metodologias foram utilizadas para a realização dos encontros, por exemplo, a utilização de slides com a utilização de imagens referentes a temática abordada. Outras estratégias utilizadas foram vídeos educativos disponibilizados via YouTube do grupo GESTAR. O grupo é composto por gestantes, com idades entre 17 e 35 anos. A maioria das participantes está em sua primeira gestação. A comunicação é realizado por meio do grupo no WhatsApp e nas demais mídias sociais.

A partir de abril de 2021 , os encontros ocorrem sobre o prisma de rodas de conversa entre moderador e convidado (especialista da área do tema do encontro), sob a participação dos demais via chat. Essa metodologia foi proposta com o intuito de favorecer a participação das usuárias e a troca de experiências entre elas.

Castiglioni et al (2020) refere que durante a realização dos grupos, observase, que as mulheres percebem a necessidade e anseiam receber informações, e ao mesmo tempo acabam tornando-se multiplicadoras do conhecimento com seus iguais, pois ao trocarem vivências e informações geram importantes fontes transformadoras de suas limitações e necessidades, adquirindo domínio sobre seu corpo e poder de decisão sobre sua gravidez.

Cardoso et al (2021) afirmam que a saúde da mulher deve ser atendida em sua totalidade, transcendendo a condição biológica de reprodutora e conferindo-se o direito de participar globalmente das decisões que envolvem sua saúde. Nesse sentido, ainda, referem que a comunicação e informação em saúde entre profissionais e gestantes devem ser priorizadas no transcurso da assistência prénatal em todo e qualquer atendimento, uma vez que a troca de informações e 
experiências pode ser a melhor forma de promover a compreensão do processo gestacional.

No decorrer deste período observa-se que as participantes estão mais seguras quanto ao processo gestacional e quanto aos cuidados que devem ter consigo e com o bebê após o nascimento. Para a maioria, as ações educativas são importantes, pois faz com que elas não fiquem com tantas dúvidas, medos e ansiedade durante a gestação e o parto.

\section{CONCLUSÃO}

A realização de grupos de forma remota tem relevância no fortalecimento de vínculos entre as gestantes, seus grupos sociais e profissionais, potencializando o cuidado da mulher e dos processos que envolvem a gestação. Fica perceptível a importância das atividades de educação em saúde, em virtude das mesmas serem utilizadas como ferramenta para promoção da saúde.

A compreensão dos aspectos do processo gestacional, puerpério e maternidade a partir dos encontros, possibilita à usuária o empoderamento frente à este momento. Assim, sugere-se que espaços como esses sejam ampliados por estudantes e profissionais para maior qualificação da atenção materno infantil.

\section{REFERÊNCIAS}

ALVES, V. T.; BEZERRA, M. M. M. Principais alterações fisiológicas e psicológicas durante o Período Gestacional. Revista Multidisciplinar e de Psicologia, v. 14, n. 49, p. 114-126, 2020.

ARRAIS, A. R.; MOURÃO, M. A.; FRAGALLE, B. O pré-natal psicológico como programa de prevenção à depressão pós-parto. Revista Saúde e Sociedade, v. 23, n. 1, p. 251-264, 2014.

CARDOSO, P. C. et al. A saúde materno-infantil no contexto da pandemia de COVID-19: evidências, recomendações e desafios. Revista Brasileira Saúde Materno Infantil, v. 21, n. 1, p. 213-220, 2021.

CASTIGLIONI, C. M. et al. Práticas de cuidado no puerpério desenvolvidas por enfermeiras em Estratégias de Saúde da Família. Revista de Enfermagem UFSM, v. 10, e50:1-19, 2020. 
GUERREIRO, E. M. et al. Ocuidado pré-natal na atenção básica de saúde sob o olhar de gestantes e enfermeiros. Revista Mineira de Enfermagem, v. 16, n. 3, p. 315-323, 2012.

NUNES, G. P. et al. Grupo de gestantes como ferramenta de instrumentalização e potencialização do cuidado. Revista de Extensão e Cultura, v. 1, n. 1, p. 1-16, 2017.

NETTO, L. A. et al. Atuação da enfermagem na conservação da saúde de mulheres em situação de violência. Revista Mineira de Enfermagem. v. 22, e-1149, 2018.

VASCONCELOS, M. I. O. et al. Intervenção Educativa em Saúde com Grupo de Gestantes: Estudantes de Enfermagem em Ação Extensionista no Interior do Ceará. Expressa Extensão. Pelotas, v.21, n.2, p. 108- 118, 2016. 